Proceeding Series of the Brazilian Society of Computational and Applied Mathematics

\title{
Sistemas Caóticos com Circuito de Chua
}

\section{Tiago Carvalho Martins ${ }^{1}$}

Faculdade de Física, Instituto de Ciências Exatas, UNIFESSPA, Marabá, PA

Resumo. Neste trabalho, o circuito de Chua é descrito em detalhes, com obtenção dos pontos de equilíbrio e das condições de estabilidade para o sistema. A partir dessas informações, é mostrado como obter um circuito de Chua caótico, para o qual é obtido o expoente de Lyapunov maximal.

Palavras-chave. Circuito de Chua, Sistemas caóticos, Memoristores.

\section{Introdução}

O circuito mostrado na Figura 1 é conhecido na literatura como "circuito de Chua", o qual é representado pelo sistema de equações diferenciais ordinárias tridimensional apresentado na equação 1 [3].

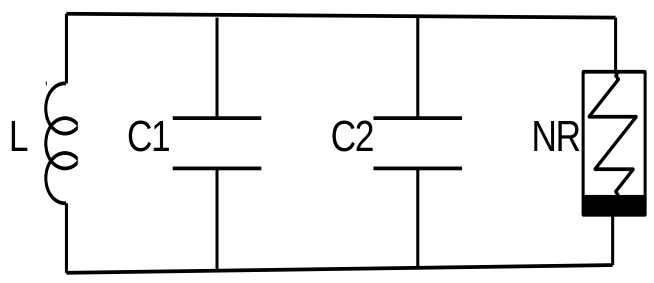

Figura 1: Circuito de Chua.

$$
\begin{aligned}
\dot{x} & =\alpha(y-x-f(x))=h_{1}(x, y, z) \\
\dot{y} & =x-y+z=h_{2}(x, y, z)
\end{aligned} \quad \alpha, \beta \in \mathbb{R}
$$

\footnotetext{
${ }^{1}$ tiagocm@unifesspa.edu.br
} 
A função $f(x)$ é dada pela expressão mostrada na equação 2 .

$$
f(x)=b x+\frac{1}{2}(a-b)(|x+1|-|x-1|) \quad a, b \in \mathbb{R}
$$

O circuito de Chua possui uma dinâmica mais complexa do que aquela exibida pelos atratores de Lorenz ou de Rössler, apresentando uma grande variedade de fenômenos os quais têm sido verificados experimentalmente em laboratório. A criptografia constitui-se em uma importante aplicação para esse tipo de circuitos [5].

O componente de circuito não-linear necessário à construção de circuitos com comportamento caótico é um dispositivo nanoeletrônico, denominado de memoristor, o qual pode ser emulado com componentes convencionais. Em 2008, um memoristor foi desenvolvido por pesquisadores da HP Labs e é conhecido como memristor da HP [3]. Em 2010, um oscilador caótico simples, utilizando um memoristor, foi obtido por Muthuswamy e Chua [4]. No mesmo ano, um atrator caótico multi-espiral foi estudado e implementado experimentalmente [1]. Em 2012, um circuito caótico baseado em memristores HP foi publicado [2].

Um sistema é dito caótico quando possui uma dependência sensível às condições iniciais, de modo que, pequenas variações nas condições iniciais alteram significativamente a trajetória do sistema. Os expoentes de Lyapunov constituem-se nas quantidades que caracterizam a taxa de separação de trajetórias infinitesimalmente próximas [maple].

As trajetórias, para um dado valor inicial, podem ser encontradas através da solução da equação 1. Esse cálculo pode ser realizado através de métodos numéricos como RungeKutta.

\section{Pontos de Equilíbrio}

Os pontos de equilíbrio de um sistema de equações diferenciais ordinárias são obtidos quando $\dot{x}=\dot{y}=\dot{z}=0$. O sistema de equações cujas soluções são os pontos de equilíbrio para o circuito de Chua é dado por

$$
\begin{array}{r}
\alpha y+(-\alpha b-\alpha) x-\alpha b+a \alpha=0, \quad z-y+x=0, \quad-\beta y=0, \quad x<-1 \\
x=\alpha y+(-a-1) \alpha x, \quad y=z-y+x, \quad z=-\beta y, \quad-1<x<1 \\
x=\alpha y+(-\alpha b-\alpha) x+\alpha b-a \alpha, \quad y=z-y+x, \quad z=-\beta y, \quad x>1 .
\end{array}
$$

A solução das equações anteriores resulta nas coordenadas $x, y$ e $z$ para os pontos de equilíbrio:

$$
\begin{array}{rlr}
x=-\frac{b-a}{b+1}, & y=0, & z=\frac{b-a}{b+1}, \quad x<-1 \\
x=0, & y=0, & z=0, \quad-1<x<1 \\
x=\frac{b-a}{b+1}, & y=0, & z=-\frac{b-a}{b+1}, \quad x>1 .
\end{array}
$$




\section{Critérios de Estabilidade}

A matriz jacobiana para o circuito de Chua pode ser obtida a partir da equação 1 , como mostrado na equação 5 .

$$
J=\left(\begin{array}{ccc}
\frac{\partial f_{1}}{\partial x} & \frac{\partial f_{1}}{\partial y} & \frac{\partial f_{1}}{\partial z} \\
\frac{\partial f_{2}}{\partial x} & \frac{\partial f_{2}}{\partial y} & \frac{\partial f_{2}}{\partial z} \\
\frac{\partial f_{3}}{\partial x} & \frac{\partial f_{3}}{\partial y} & \frac{\partial f_{3}}{\partial z}
\end{array}\right)=\left(\begin{array}{ccc}
\alpha\left(-0.5(a-b)\left(\frac{x+1}{|x+1|}-\frac{x-1}{|x-1|}\right)-b-1\right) & \alpha & 0 \\
1 & -1 & 1 \\
0 & -\beta & 0
\end{array}\right)
$$

Portanto, a jacobiana admite duas formas diferentes, ambas constantes, as quais são mostradas na equação 6. O algoritmo para o cálculo dos coeficientes de Lyapunov para o circuito de Chua deve possuir uma estrutura de decisão que permita escolher entre essas duas formas.

$$
\begin{gathered}
\left(\begin{array}{ccc}
-\alpha(b+1) & \alpha & 0 \\
1 & -1 & 1 \\
0 & -\beta & 0
\end{array}\right), \quad x<-1 \text { ou } x<-1 \\
\left(\begin{array}{ccc}
-\alpha(a+1) & \alpha & 0 \\
1 & -1 & 1 \\
0 & -\beta & 0,
\end{array}\right) \quad-1<x<1 .
\end{gathered}
$$

Portanto, para o ponto de equilíbrio localizado na origem, a equação secular é dada por

$$
\lambda^{3}+((a+1) \alpha+1) \lambda^{2}+(\beta+a \alpha) \lambda+(a+1) \alpha \beta=0 .
$$

Um ponto de equilíbrio é assintoticamente estável quando as partes reais de todos os autovalores são negativas, o que ocorre, segundo o critério de Routh-Hurwitz, quando

$$
\begin{aligned}
((a+1) \alpha+1) & >0 \\
((a+1) \alpha+1)(\beta+a \alpha)-(a+1) \alpha \beta & >0 \\
(a+1) \alpha \beta & >0 .
\end{aligned}
$$

Os parâmetros $\alpha$ e $\beta$ caracterizam componentes de circuito passivos convencionais, os quais obedecem ao princípio da reatância de Foster e, portanto, são positivos. Como $(a+1) \alpha \beta>0$, então $a>-1$, para atender à primeira e à última condição da equação anterior. Simplificando a segunda condição, tem-se que $\beta>-a \alpha((a+1) \alpha+1)$.

Repetindo a análise anterior para o caso em que os dois outros pontos de equilíbrio são assintoticamente estáveis, obtém-se: $b>-1$ e $\beta>-b \alpha((b+1) \alpha+1)$. 


\section{Resultados}

Foram calculados a trajetória e o expoente de Lyapunov maximal para os seguintes parâmetros: $\alpha=9, \beta=100 / 7, a=-8 / 7$ e $b=-5 / 7$. Quando o expoente de Lyapunov maximal é positivo o sistema apresenta comportamento caótico. Para os parâmetros escolhidos, o expoente de Lyapunov é 0.33 e, portanto, o sistema é caótico. Como $a<-1$, a origem $(0,0,0)$ é um ponto de equilíbrio instável. Os outros pontos de equilíbrio são $(-1.5,0,1.5)$ e $(1.5,0,-1.5)$. Nesse caso, a trajetória descreve o atrator de Chua com dupla-espiral, mostrado na Figura 2.

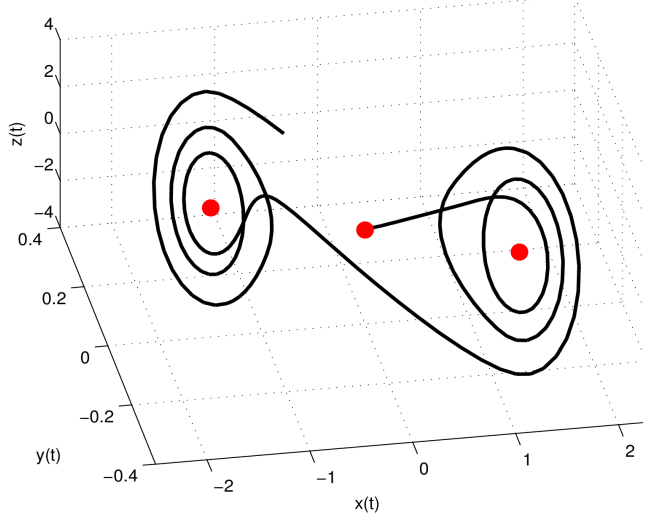

(a)

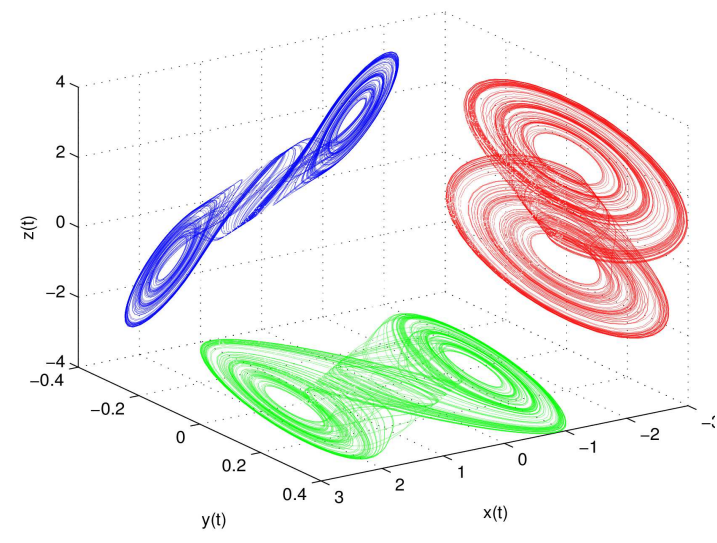

(b)

Figura 2: Atrator de Chua com dupla-espiral obtido para $\alpha=9, \beta=100 / 7, a=-8 / 7$ e $b=-5 / 7$ : (a) trajetória para $\mathrm{t}=0,15$ e (b) projeções ortogonais da trajetória para $\mathrm{t}=5$.

Foram utilizados os parâmetros $a=-1.7724, b=-0.7192, \alpha=3.8499$ e $\beta=4.4328$. Para esses valores $a<-1$, e a origem $(0,0,0)$ é um ponto de equilíbrio instável. Os outros pontos de equilíbrio são $(-3.7513,0,3.7513)$ e $(3.7513,0,-3.7513)$. Para os parâmetros escolhidos, o expoente de Lyapunov é 0.18 e, portanto, o sistema é caótico. O atrator é mostrado na Figura 3. 


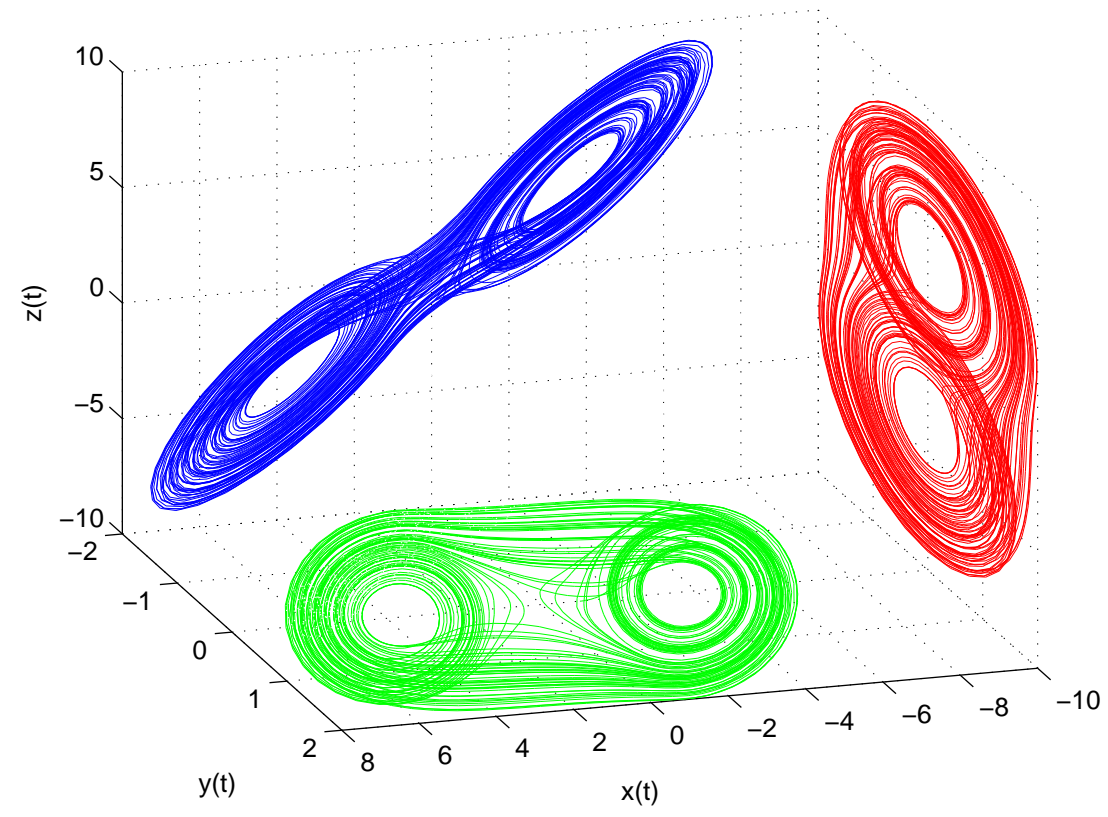

Figura 3: Projeções ortogonais da trajetória para o atrator de Chua de parâmetros: $a=-1.7724$, $b=-0.7192, \alpha=3.8499$ e $\beta=4.4328$.

\section{Conclusões}

Neste trabalho, o circuito de Chua foi descrito em detalhes. Foram obtidos os pontos de equilíbrio e as condições de estabilidade para o sistema. Foi mostrado como obter um circuito de Chua caótico, para o qual foi obtido o expoente de Lyapunov maximal, corroborando que o mesmo representa um sistema caótico.

\section{Referências}

[1] B. Bao, L. Zhu, X. Wang, Z. Liu and J. Xu, Generating multi-scroll chaotic attractor from a linear system driven by saturated function series, Communications, Circuits and Systems (ICCCAS), 2010 International Conference on, Chengdu, pp. 747-751, (2010). DOI: 10.1109/ICCCAS.2010.5581876

[2] A. Buscarino, L. Fortuna, M. Frasca, L. V. Gambuzza, A chaotic circuit based on Hewlett-Packard memristor, Chaos: Interdiscip. J. Nonlinear Sci. 22(2), 023136, (2012).

[3] S. Lynch, Dynamical Systems with Applications using MAPLE, Birkhäuser Boston, (2013). 
[4] B. Muthuswamy, L. O. Chua, Simplest Chaotic Circuit, Int. J. Bifurc. and Chaos, 20 (05), 1567-1580, (2010).

[5] A.G. Radwan, and M.E. Fouda, On the Mathematical Modeling of Memristor, Memcapacitor, and Meminductor, Springer International Publishing, (2015).

[3] D. B. Strukov, G. S. Snider, D. R. Stewart and S. R. Williams, The missing memristor found, Nature 453: 80-83, (2008). 